\title{
Single Domain Spin Manipulation by Electric Fields in Strain Coupled Artificial Multiferroic Nanostructures
}

\author{
M. Buzzi, ${ }^{1}$ R. V. Chopdekar, ${ }^{1 *}$ J. L. Hockel, ${ }^{2}$ A. Bur, ${ }^{2}$ T. Wu, ${ }^{2}$ N. Pilet, ${ }^{1}$ P. Warnicke, ${ }^{1}$ G. P. Carman, ${ }^{2}$ \\ L. J. Heyderman, ${ }^{1,3}$ and F. Nolting ${ }^{1}$ \\ ${ }^{1}$ Paul Scherrer Institut, 5232 Villigen PSI, Switzerland \\ ${ }^{2}$ Department of Mechanical and Aerospace Engineering, University of California, Los Angeles, California 90095, USA \\ ${ }^{3}$ Laboratory of Mesoscopic Systems, Department of Materials, ETH Zurich, 8093 Zurich, Switzerland
}

(Received 12 April 2013; revised manuscript received 4 June 2013; published 9 July 2013)

\begin{abstract}
We demonstrate in situ $90^{\circ}$ electric field-induced uniform magnetization rotation in single domain submicron ferromagnetic islands grown on a ferroelectric single crystal using x-ray photoemission electron microscopy. The experimental findings are well correlated with micromagnetic simulations, showing that the reorientation occurs by the strain-induced magnetoelectric interaction between the ferromagnetic nanostructures and the ferroelectric crystal. Specifically, the ferroelectric domain structure plays a key role in determining the response of the structure to the applied electric field, resulting in three strain-induced regimes of magnetization behavior for the single domain islands.
\end{abstract}

PACS numbers: 75.85.+t, 68.37.Yz, 75.75.-c, 77.55.Nv

The continuously increasing demand for data storage systems that exhibit both high speed and low energy consumption has encouraged researchers to look for novel ways of manipulating and recording information. Magnetoelectric multiferroics, materials that show both a coexistence and a coupling of magnetic and ferroelectric ordering, are considered among the most promising materials for applications in spintronic devices due to the possibility of manipulating their magnetic state through the application of an electric field [1]. However, single phase ferromagnetic-ferroelectric multiferroic materials are rare [2] and, up to now, not suitable for the development of devices due to their low ordering temperatures or lack of strong magnetoelectric coupling. One possible way of overcoming this limitation is to form a composite of two ferroic constituent materials, such as a magnetostrictive ferromagnet (FM) and a piezoelectric ferroelectric (FE), to form an artificial multiferroic [3]. Indeed electric field control of magnetization has been successfully reported for various $\mathrm{FM} / \mathrm{FE}$ continuous film heterostructures: $\mathrm{CoFe}_{2} \mathrm{O}_{4} / \mathrm{BaTiO}_{3}$ [4], $\mathrm{Fe}_{3} \mathrm{O}_{4} / \mathrm{Pb}\left(\mathrm{Mg}_{0.66} \mathrm{Nb}_{0.33}\right) \mathrm{O}_{3}-\mathrm{PbTiO}_{3}$ (PMN-PT) [5], Ni/PMN-PT [6], and CoFeB/PMN-PT [7], and it has been recently proposed $[8,9]$ that electric fieldinduced magnetization reorientations can play an important role in improving the performance of magnetic random access memories (MRAM) based on magnetic tunnel junctions [10]. At present, these devices permit facile read out of the encoded information via the tunnel magnetoresistance [11], but writing information to the device requires high magnetic fields or large current densities leading to a high energy consumption. Thus, employing magnetoelectric coupling for encoding information is of primary interest in order to build more efficient devices. Recently, an electric field-induced magnetization reversal in $\mathrm{CoFe} / \mathrm{BiFeO}_{3}$ artificial multiferroic microstructures has been reported $[12,13]$. However, the $\mathrm{CoFe}$ island is in a multidomain magnetic state and the observed magnetic reorientation is only achieved with significantly different initial and final FM domain configurations.

For reproducible switching and device reliability it is desirable to induce a reorientation between two single domain states. It has been proposed [14] that this can be achieved by manipulating the magnetic state of a single domain particle by strain-driven magnetoelectric coupling. A direct experimental proof, however, is still missing. In this work we report on the experimental observation of an electric field-induced $90^{\circ}$ uniform magnetization reorientation in single domain $\mathrm{Ni}$ elliptical nanostructures deposited on a PMN-PT ferroelectric crystal. The magnetization in each nanoisland rotates from the as-deposited in-plane easy axis defined by the shape anisotropy, to an inplane orthogonal direction induced by the converse magnetoelectric interaction generated by piezoelectric strain. The initial and final magnetization configurations are single domain as shown by $\mathrm{x}$-ray photoemission electron microscopy (X-PEEM) measurements and confirmed by micromagnetic simulations, rendering the observed phenomenon interesting for applications such as in magnetoelectric MRAMs or spin-wave magnetoelectric cells [15].

The nanoislands were prepared by electron-beam lithography and lift-off on a double-sided polished $10 \times 10 \times$ $0.5 \mathrm{~mm}^{3}$ PMN-PT (011), ferroelectric single crystal (Atom Optics Co. Ltd., Shangai, China), depositing a $50 \mathrm{~nm} \mathrm{Pt}$ buffer layer as a top contact, and $50 \mathrm{~nm}$ of Ti as a back contact. Subsequently, elliptical nanostructures were defined on top of the Pt layer using electron-beam lithography. After patterning of the electron-beam resist a $3 \mathrm{~nm}$ $\mathrm{Ti} / 10 \mathrm{~nm} \mathrm{Ni} / 2 \mathrm{~nm}$ Pt trilayer was deposited by electronbeam evaporation, and the following lift-off resulted in isolated magnetic nanoislands [Fig. 1(a)]. The composition of PMN-PT used in this work is close to the morphotrophic 


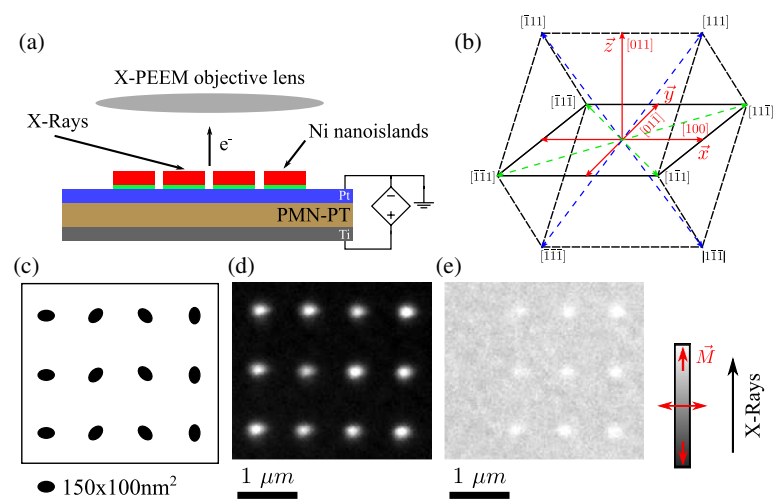

FIG. 1 (color online). (a) Schematic of the experiment (not to scale). (b) Sketch of the rhombohedral crystal structure highlighting out-of-plane (blue) and in-plane (green) equivalent polar axes. (c) Schematic of the imaged area with $150 \times 100 \mathrm{~nm}^{2}$ nanoislands in four different orientations, with the ellipse long axis at $90^{\circ},-45^{\circ}, 45^{\circ}$, and $0^{\circ}$ with respect to the $\mathrm{x}$-ray direction. (d) X-PEEM elemental contrast image, and corresponding (e) XMCD image at the Ni $L_{3}$ edge after magnetic saturation of the sample.

phase boundary region of the niobate-titanate alloy [16] and the crystal structure of the PMN-PT is rhombohedral with a ferroelectric polar axis along the $\langle 111\rangle$ directions. Figure 1(b) shows a sketch of the crystal structure with [011] being the substrate normal direction.

The magnetic configuration of the nanostructures has been investigated by X-PEEM at the Surface/Interface Microscopy (SIM) beam line of the Swiss Light Source [17]. Imaging of the magnetic state of the nanostructures was performed employing $\mathrm{x}$-ray magnetic circular dichroism (XMCD) tuning the incoming photon energy to the $\mathrm{Ni} L_{3}$ edge $(\approx 852 \mathrm{eV})$ and acquiring images with opposite circular polarization directions $\left(c_{+}\right.$and $\left.c_{-}\right)$ [18]. The XMCD image, calculated as the asymmetry $\left(c_{+}-c_{-}\right) /\left(c_{+}+c_{-}\right)$, emphasizes the magnetic contrast, which is proportional to the scalar product $\vec{M}(\vec{r}) \cdot \vec{k}$, where $\vec{k}$ is the propagation vector of the incoming $\mathrm{x}$ rays [19]. In other words, the XMCD image shows black or white contrast in areas where magnetic moments have a component parallel or antiparallel to $\vec{k}$. The Ni elemental contrast image is defined as a Ni $L_{3}$ edge image normalized to an image taken at a preedge energy, and highlights the presence of the isolated Ni nanostructures on the continuous Pt electrode. To investigate the ferroelectric configuration of the sample, piezoresponse force microscopy (PFM) measurements have been performed at the NanoXAS beam line of the Swiss Light Source [20].

To assess the magnetic configuration of the magnetic nanostructures, we have performed investigations with no electric field applied to the sample. Figure 1 shows (c) a sketch of a 4 by 3 array of $150 \times 100 \mathrm{~nm}^{2}$ ellipses, (d) the corresponding Ni elemental contrast and (e) the XMCD contrast image. The sample is imaged after applying a saturating magnetic field pulse of $\approx 0.3 \mathrm{~T}$ along the $\mathrm{x}$-ray direction. The elemental contrast image, Fig. 1(d) shows that Ni nanostructures are well isolated and can be clearly distinguished. If we assume that shape anisotropy is the dominant contribution to the overall magnetic anisotropy of the islands, the left column of the array of structures should have an expected magnetic easy-axis perpendicular to the $\mathrm{x}$-ray direction, whereas the right column should have magnetization oriented parallel to the $\mathrm{x}$-ray direction. Indeed, the islands with the long axis perpendicular to the $\mathrm{x}$-ray propagation direction give in the $\mathrm{XMCD}$ image a gray contrast not distinguishable from the background that indicates that their magnetization is perpendicular to $\vec{k}$. In comparison, the ellipses elongated along the x-ray $\vec{k}$ vector appear white since their magnetization is uniformly aligned with $\vec{k}$ and thus have maximum $\mathrm{XMCD}$. Thus, for $10 \mathrm{~nm}$ thick Ni $150 \times 100 \mathrm{~nm}^{2}$ islands the long axis then determines the easy magnetization direction of the nanostructures due to the shape anisotropy.

To investigate the magnetoelectric interaction effects we have imaged the magnetization configuration of an array of $200 \times 100 \mathrm{~nm}^{2}$ ellipses while varying the applied electric field from 0 to $0.27 \mathrm{MV} \mathrm{m}^{-1}$ as shown in Fig. 2(a), and plot the quantitative XMCD intensity as a function of the applied electric field in Fig. 2(b) for all the ellipses showing a permanent change during the electric field sweep. Before the experiment, the sample is initially poled at $-0.4 \mathrm{MV} \mathrm{m}^{-1}$ and magnetically randomized with a commercial ac demagnetizer. All the ellipses in the array have their long axis parallel to the [011] direction of the FE crystal and parallel to the $\mathrm{x}$-ray propagation direction. The initial randomized magnetic configuration at $0 \mathrm{MV} \mathrm{m}^{-1}$ indicates that the islands appear uniformly magnetized either parallel (black) or antiparallel (white) to the x-ray

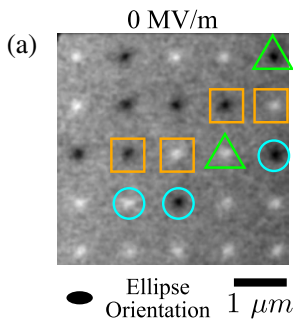

(b)

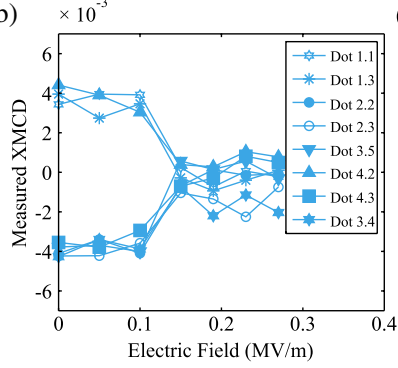

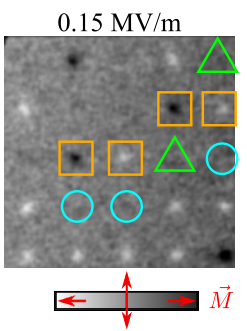

(c)

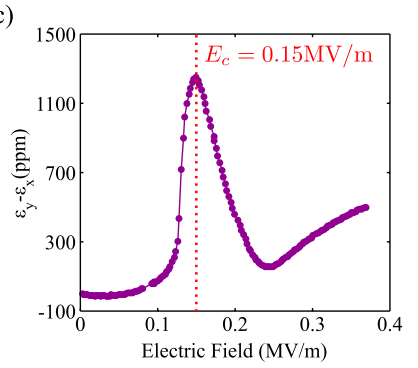

FIG. 2 (color online). (a) Sequence of successive XMCD images of $200 \times 100 \mathrm{~nm}^{2}$ ellipse array recorded at the Ni $L_{3}$ edge at different applied electric fields. (b) XMCD contrast as a function of the applied electric field curve for selected dots labeled in (row, column) format in panel (a). (c) In-plane strain vs applied electric field curve measured with a strain gauge fixed on the sample after poling. 
propagation direction. We find that by increasing the applied electric field no changes are seen until the ferroelectric coercive electric field, $E_{c}=0.15 \mathrm{MV} \mathrm{m}^{-1}$, is reached [21]. As we approach $E_{c}$, three different types of magnetic behavior can be observed, as highlighted in Fig. 2. (i) For the ellipses circled in blue, the magnetic contrast from the nanoislands drops from the original value to zero, indicating that the magnetization of the ellipses has rotated away from the long axis and now lies parallel to the ellipse short axis. By increasing the electric field up to $0.27 \mathrm{MV} \mathrm{m}^{-1}$, no changes in the magnetic configuration are observed. (ii) The ellipses highlighted with green triangles show a different behavior. After undergoing the $90^{\circ}$ reorientation at $E_{c}$, when the applied electric field is increased further the magnetization relaxes back to one of the two equivalent directions parallel to the shape anisotropy easy axis. (iii) Finally, ellipses highlighted with orange squares do not show any change for any value of the applied electric field.

It can be excluded that the observed reduction of magnetic contrast which we observe at $E_{c}$ is due to an imaging artifact as a result of the applied electric field since the orange-highlighted dots in the array do not show any change for any of the applied electric fields. Figure 2(b) shows that the drop in magnetic contrast observed when the applied electric field is equal to $E_{c}$ can be correlated to the corresponding increase in the induced strain in the Pt film and magnetic nanostructures shown in Fig. 2(c). We propose that the observed electric field-induced magnetization reorientation can be explained by strain-mediated magnetoelectric coupling which causes the magnetization to reorient as a result of the competition of shape anisotropy and magnetoelastic contributions induced by the ferroelectric distortions. However, since the ellipses are of uniform size and shape [22], and give the same magnitude of $\mathrm{XMCD}$ in the randomized state at $0 \mathrm{MV} \mathrm{m}^{-1}$, we can speculate that the nanostructures should show identical magnetic behavior. Thus, the observation of these three different types of behavior suggests that there are three different regimes of strain coupling. This we can explain, as follows, with a more detailed analysis which takes into account how the strain is generated in the magnetic nanostructures as the ferroelectric polarization is reversed.

As shown in Fig. 1(b) the FE polarization can be oriented along any of the 8 possible directions: 4 in the $(01 \overline{1})$ plane (blue dashed lines, FE axis with a component along the surface normal) and 4 in the (011) plane (green dashed lines, FE axis fully in the plane of the sample). As already reported elsewhere [21] the polarization reversal after poling along the [011] direction happens with two subsequent $71^{\circ}-109^{\circ}$ rotations starting from the equivalent two out-of-plane diagonals pointing in the poling direction. When the applied electric field is equal to $E_{c}$ the polarization rotates to lie along one of the four possible in-plane orientations, causing a strong expansion of the FE structure along the [011] and [100] directions. By further increasing the electric field the FE polarization will undergo the second $71^{\circ}-109^{\circ}$ rotation to an opposite out-of-plane diagonal completing the reversal and reducing the structural distortion to the value solely caused by electrostriction due to the applied electric field. If the electric field is then removed, the FE axis remains along the reversed [111] direction and the initial in-plane lattice dimensions are recovered.

Let us first analyze how the magnetic energy balance of a nanoisland changes when the ferroelectric polarization is reversed. Figure 2(c) shows the in-plane strain vs electric field curve measured by a strain gauge fixed to the Pt top contact on the PMN-PT crystal while switching the ferroelectric polarization after poling along the [011] direction. This situation corresponds to a nanoisland grown on an out-of-plane poled FE crystal. Since the Ni ellipses are polycrystalline, no magnetocrystalline anisotropy contributions are expected and the two competing terms are the shape anisotropy and the magnetoelastic energy due to the applied strain by the piezoelectric. We can then express the magnetoelastic energy term for $\mathrm{Ni}$ as $U_{\mathrm{me}}=$ $-(3 / 2) \lambda_{p} Y\left(\varepsilon_{01 \overline{1}}-\varepsilon_{100}\right) \cos ^{2}\left(\theta_{01 \overline{1}}\right)$ where $\lambda_{p}$ is the magnetostriction coefficient for polycrystalline $\mathrm{Ni}, Y$ is the Young's modulus of nickel, $\left(\varepsilon_{01 \overline{1}}-\varepsilon_{100}\right)$ the applied strain by the piezoelectric and $\theta_{01 \overline{1}}$ the angle between the magnetization and the [011] direction of the FE. For an ellipse with the long axis oriented along [011] , positive values of $\left(\varepsilon_{01 \overline{1}}-\varepsilon_{100}\right)$ will compete with the shape anisotropy favoring a reorientation of the magnetization to the [100]. According to finite element magnetostatic calculations using the NMAG code [23], for the $200 \times 100 \mathrm{~nm}^{2} \mathrm{Ni}$ nanostructures shown in Fig. 2 with a 2:1 ratio between the long and short in plane axes, the magnetostatic energy barrier to be overcome for inducing the rotation to the short axis is $\approx 10 \mathrm{~kJ} \mathrm{~m}^{-3}$. When the polarization of the $\mathrm{FE}$ is switched, the quantity $\left(\varepsilon_{01 \overline{1}}-\varepsilon_{100}\right)$ jumps to $\approx 1300 \mathrm{ppm}$. This generates a Ni magnetoelastic contribution of about $15 \mathrm{~kJ} \mathrm{~m}^{-3}$ [24], which is sufficient to overcome the magnetostatic energy barrier and to induce the $90^{\circ}$ magnetization reorientation.

The three different behaviors of the nanoislands described above can be understood taking into account the underlying FE domain structure of the crystal when the Ni structures are deposited. This is supported by PFM measurements taken on an identical PMN-PT crystal, which cannot be performed on the fully fabricated sample used for the PEEM experiment due to the presence of the Pt top electrode preventing retrieval of the PFM contrast. Figure 3(a) shows the presence of alternating in-plane and out-of-plane domains, with FE axis along any of the eight $\langle 111\rangle$ type directions. The domain length scale is $\approx 1 \mu \mathrm{m}$ which is comparable to the ellipse spacing in the array of Fig. 2 and is $\approx 5$ times larger than the ellipse size. It is then reasonable to assume that a significant amount of nanoislands lie on a single FE domain, although adjacent ellipses may be on domains with different FE orientations or even on top of a FE domain boundary between different FE orientations. Since the Ni ellipses are polycrystalline, the zero value of the $\mathrm{Ni}$ internal strain depends on the substrate distortion at the deposition time. Let us first consider the case presented in Fig. 3(b) of a nanostructure 


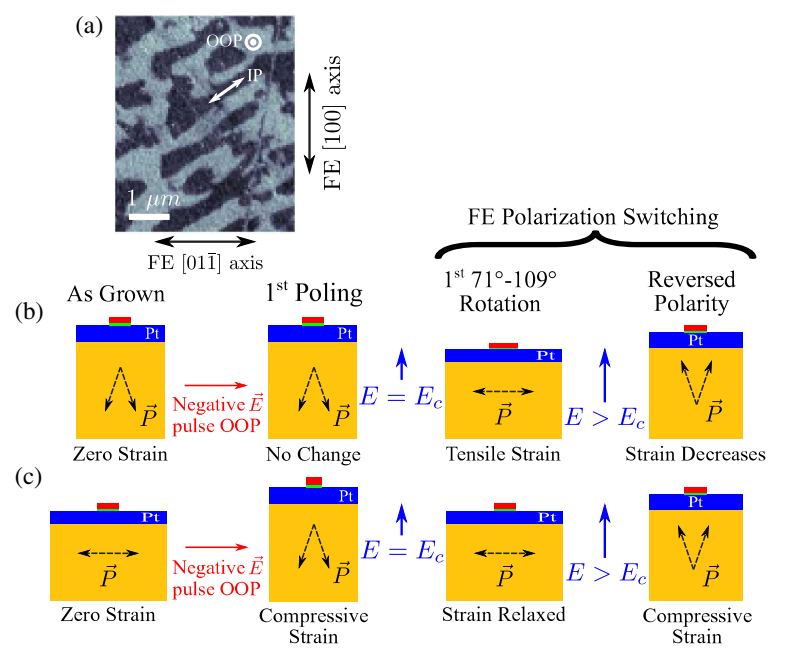

FIG. 3 (color online). (a) PFM image of the out-of-plane piezoelectric response of the PMN-PT crystal. FE domain orientations are indicated by the out-of-plane (OOP) and in-plane (IP) white arrows in the image. (b), (c) Strain behavior, during FE polarization reversal for a dot deposited on an out-of-plane and in-plane polarized FE domain, respectively.

deposited on an out-of-plane FE domain. In this case, the $\mathrm{Ni}$ is deposited on an area of the FE in which the polarization is already pointing out of the (011) plane and the first macroscopic poling, applying a field along [011] before the measurement, will not modify the distortion of the structure. When $E \approx E_{c}$, the first $71^{\circ} / 109^{\circ} \mathrm{FE}$ polarization rotation to the in-plane polar axes will cause the FE structure to expand along the main in-plane directions. This generates a tensile strain in the Ni nanostructure which as explained above triggers the magnetization reorientation. This single-step magnetization reorientation is the behavior shown by the blue-circled ellipses in Fig. 2(a). In Fig. 3(c), we consider a nanoisland deposited on an inplane poled domain. The $\mathrm{Ni}$ is deposited on an area polarized in the (011) plane and the FE crystal underneath the ellipse is already elongated along the [100] and [011] directions. At the first poling along the [011] direction, the polarization in this domain will rotate out-of-plane, causing a strong compressive strain in the Ni nanostructure which will act to favor further the easy axis induced by the shape anisotropy. Cycling the electric field will only relax the effective compressive strain in the magnetic ellipse and no reorientation will happen regardless of the applied electric field, which explains the magnetic behavior shown by the ellipses marked by an orange square. Finally, let us now consider the case of the ellipses marked by a green triangle in which the magnetization relaxes back to the shape anisotropy easy axis for $E>E_{c}$. After the strain maximum at $E_{c}$, the strain starts to decrease down to $\approx 200 \mathrm{ppm}$, as shown in Fig. 2(c). In the case of the blue-circled ellipses the magnetoelastic contribution is sufficient to keep the magnetization perpendicular to the shape anisotropy induced easy axis. However, if the local strain of the nanostructure is lower, for example because the nanoisland is lying on the boundary between two different FE domains, the strain-mediated magnetoelectric coupling will be weaker and at $0.27 \mathrm{MV} \mathrm{m}^{-1}$ the magnetoelastic contribution might not be strong enough to prevent the relaxation back towards the shape anisotropy easy axis [25].

To confirm the proposed interpretation we have carried out micromagnetic simulations using the NMAG code assuming a uniformly applied strain to the $200 \times 100 \mathrm{~nm}^{2}$ ellipse elongated along the [011] direction of the ferroelectric. Islands grown on both out-of-plane and in-plane ferroelectric domains have been simulated. The initial spin configuration of the system at $0 \mathrm{MV} \mathrm{m}^{-1}$ is achieved by relaxing the magnetic configuration starting from a randomly magnetized state in the absence of magnetoelastic anisotropy and applied magnetic field. As indicated by the X-PEEM measurement, the obtained equilibrium magnetization configuration, shown in Fig. 4(a) upper left panel, is single domain and uniformly magnetized along the shape anisotropy-induced easy axis. The experimental electric field effect is simulated by varying the anisotropy constant according to the corresponding strain value for the electric field derived from Fig. 2(c). The behavior of the magnetization parallel to the ellipse long axis as a function of the strain is then obtained and compared with the measured XMCD contrast. In order to match the experimental observations we have tuned the $\mathrm{Ni}$ saturation magnetization $M_{s}$ to $0.33 \mathrm{~T}$ ( $\approx 55 \%$ of the bulk value). We have carried out $\mathrm{x}$-ray absorption measurements on individual nanostructures (not shown) indicating that no oxidation layer is present at the surface of the nanostructures. Even though we cannot exclude some residual oxidation at the nanoislands edges, we believe that the reduction of $M_{s}$ needed in the simulations is caused by an underestimated strain value due to approximating the local strain with a macroscopically averaged strain gauge value. Figure 4(a) shows the evolution of the simulated magnetic configuration from $0 \mathrm{MV} \mathrm{m}^{-1}$ applied electric field to $0.15,0.27 \mathrm{MV} \mathrm{m}^{-1}$ and then back to $0 \mathrm{MV} \mathrm{m}^{-1}$ for an ellipse grown on an outof-plane FE domain. The simulation confirms the experimentally observed $90^{\circ}$ uniform magnetization rotation that occurs at $0.15 \mathrm{MV} \mathrm{m}^{-1}$ from the shape anisotropy-induced easy axis to the one induced by the strain-mediated magnetoelectric interaction. The magnetization at $0.27 \mathrm{MV} \mathrm{m}^{-1}$ reverts back towards the original direction but the angular change is only $\approx 7^{\circ}$ which is within the noise of our XMCD

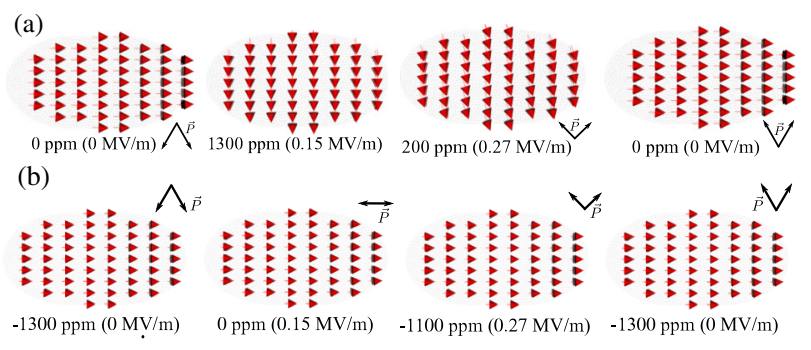

FIG. 4 (color online). Simulated magnetization profiles at 0 , $0.15,0.27$, and back to $0 \mathrm{MV} \mathrm{m}^{-1}$ for a nanostructure deposited on (a) an out-of-plane (OOP) polarized FE domain and (b) on an inplane (IP) polarized FE domain. Arrows schematically indicate the ferroelectric polarization directions of the PMN-PT crystal. 
measurement. The change in applied electric field from 0.15 to $0.27 \mathrm{MV} \mathrm{m}^{-1}$ reduces the strain to a minimum [Fig. 2(c)] and, at this point, any variation in the strain behavior, caused for example by the presence of a FE domain wall or defect, could cause the magnetization to relax back completely to the shape anisotropy-dictated easy axis. Finally, when the field is brought back to $0 \mathrm{MV} \mathrm{m}^{-1}$ the magnetization relaxes back to the original configuration. Figure 4(b) shows the results of the simulation for a nanoellipse grown on an in-plane FE domain. In this case, the only effect of the magnetoelastic interaction is to further stabilize the already easy direction induced by the shape anisotropy. As a consequence, a magnetic reorientation does not take place.

In conclusion, using X-PEEM we have demonstrated the possibility of inducing a $90^{\circ}$ uniform magnetization rotation due to an electric field in arrays of strain coupled artificial multiferroic nanostructures. Thanks to the hysteretic strain dynamics of the FE material [14], the effect can be nonvolatile and reversible constituting an important step towards the realization of a magnetoelectric MRAM cell. We have found that the multidomain structure of the FE single crystal leads to a complex strain-mediated magnetoelectric coupling. This suggests that realizing the full magnetoelectric stack at the nanoscale in order to achieve a single domain configuration in the FE as well as the FM is of primary importance not only for fulfilling large scale integration requirements but also for the achievement of reliable magnetization manipulation by an electric field. This would pave the way towards magnetoelectric-MRAM devices containing an artificial multiferroic film stack with low power consumption and high switching reliability.

The authors would like to thank C. A.F. Vaz and M. Kläui for fruitful discussions and Jörg Raabe and Juri Honegger for technical support. Part of this work was performed at the Swiss Light Source, Paul Scherrer Institute, Villigen, Switzerland. This work was partially funded by EU's 7th Framework Program IFOX (NMP3LA-2010 246102) and NSF Nanosystems Engineering Research Center for Translational Applications of Nanoscale Multiferroic Systems (TANMS) Cooperative Agreement Award EEC-1160504.

*rajesh.chopdekar@psi.ch

[1] M. Fiebig, J. Phys. D 38, R123 (2005).

[2] N. A. Hill, J. Phys. Chem. B 104, 6694 (2000).

[3] C. A. F. Vaz, J. Phys. Condens. Matter 24, 333201 (2012).

[4] H. Zheng, J. Wang, S. E. Lofland, Z. Ma, L. MohaddesArdabili, T. Zhao, L. Salamanca-Riba, S. R. Shinde, S. B. Ogale, F. Bai, D. Viehland, Y. Jia, D. G. Schlom, M. Wuttig, A. Roytburd, and R. Ramesh, Science 303, 661 (2004).

[5] M. Liu, O. Obi, J. Lou, Y. Chen, Z. Cai, S. Stoute, M. Espanol, M. Lew, X. Situ, K. S. Ziemer, V. G. Harris, and N. X. Sun, Adv. Funct. Mater. 19, 1826 (2009).
[6] T. Wu, A. Bur, P. Zhao, K. P. Mohanchandra, K. Wong, K. L. Wang, C. S. Lynch, and G.P. Carman, Appl. Phys. Lett. 98, 012504 (2011).

[7] S. Zhang, Y. G. Zhao, P. S. Li, J. J. Yang, S. Rizwan, J. X. Zhang, J. Seidel, T. L. Qu, Y. J. Yang, Z. L. Luo, Q. He, T. Zou, Q. P. Chen, J. W. Wang, L. F. Yang, Y. Sun, Y.Z. Wu, X. Xiao, X. F. Jin, J. Huang, C. Gao, X. F. Han, and R. Ramesh, Phys. Rev. Lett. 108, 137203 (2012).

[8] M. Bibes and A. Barthelemy, Nat. Mater. 7, 425 (2008).

[9] J.-M. Hu and C. W. Nan, Phys. Rev. B 80, 224416 (2009).

[10] J. Åkerman, Science 308, 508 (2005).

[11] S. Ikeda, J. Hayakawa, Y. M. Lee, F. Matsukura, Y. Ohno, T. Hanyu, and H. Ohno, IEEE Trans. Electron Devices 54, 991 (2007).

[12] J. T. Heron, M. Trassin, K. Ashraf, M. Gajek, Q. He, S. Y. Yang, D. E. Nikonov, Y.-H. Chu, S. Salahuddin, and R. Ramesh, Phys. Rev. Lett. 107, 217202 (2011).

[13] Y.-H. Chu, L. W. Martin, M. B. Holcomb, M. Gajek, S.-J. Han, Q. He, N. Balke, C.-H. Yang, D. Lee, W. Hu, Q. Zhan, P.-L. Yang, A. Fraile-Rodríguez, A. Scholl, S. X. Wang, and R. Ramesh, Nat. Mater. 7, 678 (2008).

[14] T. Wu, A. Bur, K. Wong, P. Zhao, C. S. Lynch, P. K. Amiri, K. L. Wang, and G.P. Carman, Appl. Phys. Lett. 98, 262504 (2011).

[15] A. Khitun, M. Bao, and K. Wang, IEEE Trans. Magn. 44, 2141 (2008).

[16] S. Choi, T. Shrout, S. Jang, and A. Bhalla, Mater. Lett. 8, 253 (1989).

[17] U. Flechsig, F. Nolting, A. Fraile-Rodríguez, J. Krempasky, C. Quitmann, T. Schmidt, S. Spielmann, and D. Zimoch, AIP Conf. Proc. 1234, 319 (2010).

[18] J. Stohr, Y. Wu, B. D. Hermsmeier, M. G. Samant, G. R. Harp, S. Koranda, D. Dunham, and B. P. Tonner, Science 259, 658 (1993).

[19] L. L. Guyader, A. Kleibert, A. Fraile-Rodríguez, S. E. Moussaoui, A. Balan, M. Buzzi, J. Raabe, and F. Nolting, J. Electron Spectrosc. Relat. Phenom. 185, 371 (2012).

[20] N. Pilet, J. Raabe, S. E. Stevenson, S. Romer, L. Bernard, C. R. McNeill, R. H. Fink, H. J. Hug, and C. Quitmann, Nanotechnology 23, 475708 (2012).

[21] T. Wu, P. Zhao, M. Bao, A. Bur, J. L. Hockel, K. Wong, K. P. Mohanchandra, C.S. Lynch, and G.P. Carman, J. Appl. Phys. 109, 124101 (2011).

[22] SEM measurements (not shown) indicate that the different nanoislands have similar microstructure and that the variation in aspect ratio of the nanoislands is less than $10 \%$.

[23] T. Fischbacher, M. Franchin, G. Bordignon, and $\mathrm{H}$. Fangohr, IEEE Trans. Magn. 43, 2896 (2007).

[24] $U_{\mathrm{me}}$ can be rewritten as $U_{\mathrm{me}}=K_{1} \sin ^{2} \theta_{01 \overline{1}}$ with $K_{1}=$ $(3 / 2) \lambda_{p} Y\left(\varepsilon_{01 \overline{1}}-\varepsilon_{100}\right)$. A value of $K_{1} \approx 15 \mathrm{kJm}^{-3}$ is obtained substituting the peak strain change of $1300 \mathrm{ppm}$ and $\mathrm{Ni}$ tabulated values (R. Cullity et al., Introduction to Magnetic Materials): $\lambda_{p}=3.4 \times 10^{-5}, Y=220 \mathrm{GPa}$.

[25] This interpretation holds also for the utmost bottom right element in Fig. 2(a) with a further consideration. Since ferroelectric domains with opposite polarization can nucleate already at electric fields lower than $E_{c}$, in this case the polarization reversal is locally completed at a lower field and the observed $180^{\circ}$ reorientation can be explained to be a relaxation from an early $90^{\circ}$ reorientation. 